\title{
La formación de maestros y la promoción de la competencia comunicativa de alumnos sordos
}

\author{
Teachers' education and the promotion of communicative competence of \\ deaf students
}

A formação de professores e a promoção da competência comunicativa de alunos surdos

Marta Gràcia

Profesora doctora en la Universidad de Barcelona, Barcelona, España.

mgraciag@ub.edu

ORCID - http://orcid.org/0000-0003-1280-4578

Cristina Broglia Feitosa de Lacerda

Profesora doctora em la Universidade Federal de São Carlos, São Carlos, São Paulo, Brasil.

cbflacerda@gmail.com

ORCID - http://orcid.org/0000-0002-3250-1374

Recebido em 29 de julho 2020

Aprovado em 12 de agosto de 2020

Publicado em 17 de novembro de 2020

\section{RESUMEN}

La mejora de la educación de alumnos sordos en contextos escolares inclusivos requiere la formación de maestros en relación con sus estrategias para promover la competencia comunicativa de sus alumnos. El objetivo de la investigación es mejorar las estrategias de una maestra de una escuela inclusiva de Brasil a través de una formación docente con el fin de promover la competencia comunicativa de sus alumnos. Los participantes son la maestra bilingüe y tres alumnos sordos de educación infantil. Los instrumentos utilizados son: 1) la aplicación digital EVALOE-SSD para la formación, y 2) un cuestionario de valoración final. Durante cuatro meses la maestra utilizó la aplicación para autoevaluar sus clases, tomar decisiones, e introducir cambios en su práctica docente y al final respondió al cuestionario de valoración final. Durante el mismo período, el equipo de investigación realizó tres observaciones en el aula y las evaluó con la misma aplicación. Los resultados indican que la formación supone un reto para la maestra, así como mejoras en su práctica docente y en la competencia comunicativa de los alumnos en lengua de signos. Se derivan implicaciones para la formación inicial de maestras de alumnos sordos.

Palabras clave: Formación de maestros; competencia comunicativa; educación de sordos.

\section{ABSTRACT}

The improvement of deaf students' education in the inclusive school context requires the teacher's formation regarding strategies to promote the communicative competence of their students. The objective of de research is to improve the strategies of a teacher in an inclusive school in Brazil through teacher training seeking to promote the communication competence of her studentes. The participants are the three deaf students 
in kindergarten. The instruments used are 1) a digital app EVALOE-SSD for the formation, and 2) a final assessment questionnarie. During four months the teacher used the app to self-evaluate her classes, make decisions and introduce changes in her teaching practices. During the same period, the investigation team made three classroom observations and evaluated with the same app. The results show that the formation was challenging to the teacher, with improvements in her teaching practices and in the communicative competence of the deaf children in sign language. There were implications pointed out about the initial formation of bilingual teachers for deaf.

Keywords: Teacher education; communicative competence; deaf education.

\section{RESUMO}

A melhoria da educação de alunos surdos em contextos escolares inclusivos demanda a formação de professores no que se refere a estratégias para promover a competência comunicativa de seus alunos. O objetivo desta pesquisa é melhorar as estratégias de ensino de uma professora de uma escola inclusiva no Brasil por meio de uma formação docente buscando promover a competência comunicativa de seus alunos. Os participantes são a professora bilíngue e três alunos surdos da educação infantil. Os instrumentos utilizados foram: 1) um aplicativo digital EVALOE-SSD para a formação, e 2) um questionário de avaliação final. Durante quatro meses a professora utilizou o aplicativo para autoavaliar suas aulas, tomar decisões, e introduzir mudanças em sua prática docente e ao final respondeu ao questionário de avaliação $\mathrm{N}$ mesmo período, a equipe de pesquisadores realizou três observações nas aulas e as avaliou com o mesmo aplicativo. Os resultados indicam que a formação foi desafiadora para a professora, com melhorias em sua prática docente e na competência comunicativa dos alunos surdos em língua de sinais. Foram apontadas implicações para a formação inicial de professores bilíngues de surdos.

Palavras-chave: Formação de professores; competência comunicativa; educação de surdos.

\section{Introducción}

Conseguir que los alumnos alcancen una buena competencia comunicativa oral (hablar y escuchar) en el contexto escolar, supone que esta sea considerada por los docentes un objetivo de enseñanza transversal y que se posibilite su desarrollo, fundamentalmente, mediante la creación de ambientes ricos en los cuales los aprendices participen en interacciones lingüísticas variadas y de calidad (HAMRE et al. 2014; HOWES et al., 2008). Sin embargo, no siempre los docentes tienen incorporadas las estrategias educativas necesarias para promover en sus alumnos el desarrollo de esta competencia.

Las escuelas constituyen espacios de relaciones interpersonales que pueden favorecer el desarrollo comunicativo y lingüístico cuando las interacciones lingüísticas son de calidad y el docente usa determinadas estrategias educativas (JONES, 2017; PIASTA et al. 2012; VAN DER VEEN, et al., 2017). Los interlocutores contribuyen a crear 
interacciones que redundan en el desarrollo de la competencia comunicativa que a su vez contribuyen a los aprendizajes escolares (CLARKE et al. 2016; MERCER, 2010).

Por otro lado, diferentes estudios muestran discrepancias entre las creencias sobre la lengua y las acciones lingüísticas en maestros en formación o entre cómo conceptualizan la oralidad los maestros y su evaluación en las escuelas (MERCER; WARWICK; AHMED, 2017). Todo ello pone de relieve que es necesario en la formación inicial y en el desarrollo profesional de maestros que estos amplíen sus conocimientos y reflexionen sobre sus prácticas docentes con relación a la oralidad y la interacción, para poder tomar decisiones apropiadas que la promuevan. Mediante la reflexión, pueden hacer evidentes muchos aspectos subjetivos implícitos de los cuales no siempre son conscientes y que revisten de gran importancia en su actuación profesional (KORTHAGEN, 2010).

La necesidad de evaluar la competencia comunicativa y los procesos interactivos que conducen a ella ha llevado al desarrollo de diferentes tipos de herramientas. Autora y colaboradores desarrollaron la Escala de Valoración de la Lengua Oral en contexto Escolar - EVALOE (GRÀCIA et al., 2015a). Esta conceptualiza las aulas como espacios comunicativos en los que se reflexiona y enseña la lengua oral, constituyendo esta una herramienta clave en el aprendizaje de contenidos vinculados con todas las áreas del currículum (GRÀCIA; VEGA; GALVÁN-BOVAIRA 2015b; GRÀCIA; GALVÁN-BOVAIRA; SÁNCHEZ-CANO, 2017; VAN DE POL, MERCER, VOLMAN, 2019). Fue diseñada con el objetivo de proporcionar a los maestros y profesionales del desarrollo del lenguaje (logopedas, psicopedagogos...) un recurso no solo para la evaluación de las habilidades y estrategias utilizadas por los docentes para promover el desarrollo de la competencia oral en el aula, sino también la interacción entre estos y los aprendices y sus acciones lingüísticas.

Recientemente herramientas analógicas, como la citada anteriormente, han dado paso a instrumentos digitales como recursos fundamentales en la formación del profesorado. Investigaciones como las llevadas a cabo por Gràcia et al. (2020b, o Silva Quiroz (2012) contemplan el uso de la tecnología para la reflexión sobre su propia práctica y a la introducción de mejoras. Por su parte, los Sistemas de Soporte a la toma de Decisiones - SSD son soluciones digitales que se pueden utilizar para apoyar la toma de decisiones complejas y la resolución de problemas (SHIM et al. 2002). En el ámbito educativo, la integración de los SSD es muy reciente y todavía representa un reto importante. 
http://dx.doi.org/10.5902/1984686X48495

La investigación que se presenta enfatiza la utilidad de los SSD en el ámbito educativo y, concretamente, la intención de empoderar (empower) a los maestros y ayudarles en la toma de decisiones respecto a su práctica educativa, sus estrategias discursivas y la gestión de la comunicación, así como contribuir al desarrollo de la competencia lingüística de los alumnos de educación infantil y de primaria (GRÀCIA, 2018; GRÀCIA et al., 2020b).

A partir de la versión inicial de la EVALOE como instrumento de observación se creó un instrumento de autoevaluación y de toma de decisiones: la Escala de Valoración de la enseñanza de la Lengua Oral en contexto Escolar - Sistema de Soporte a la toma de Decisiones - EVALOE-SSD (GRÀCIA et al., 2018), mediante un formulario online (Google Forms) (GRÀCIA et al., 2020a). Esta primera versión de la EVALOE-SSD autoaplicada se validó con un conjunto de seis maestras de escuelas de tipología diversa. A partir de esta primera versión autoaplicada se elaboró la versión digital de la EVALOE-SSD (GRÀCIA et al., 2018), que incorpora mejoras sustanciales, siendo una de ellas que una de las versiones está adaptada a situaciones de enseñanza de lengua de signos en contexto de escuela ordinaria (LACERDA et al., 2018; 2020) y además se encuentra en cuatro lenguas (catalán, español, inglés y portugués).

La idea del funcionamiento de la herramienta plantea que las maestras participantes autoevalúen las sesiones de clase de cualquier asignatura, una vez por semana aproximadamente. Con esta versión de la aplicación digital revisada se realizó la segunda recogida de datos (GRÀCIA, 2018; GRÀCIA et al., 2020a) con resultados alentadores.

En Brasil, la inclusión escolar de alumnos sordos prevé la presencia y acompañamiento del intérprete de lengua de signos a partir de la Enseñanza Fundamental II (Decreto 5626/2005) (BRASIL, 2005), considerando que en esta etapa de enseñanza la fluencia en lengua de signos ya estará bastante desarrollada, y que los alumnos sordos podrán aprender los contenidos escolares gracias a los procesos de traducción (LACERDA, 2009; LACERDA; LODI., 2009). La adquisición y el desarrollo del lenguaje se produce gracias a la participación en situaciones de interacción con otros hablantes de modo contextual, natural y gradual, también en los niños sordos, en cuyo caso la lengua de interacción debería ser la lengua de signos y además la oral, si en el contexto hay personas oyentes.

Cabe resaltar que la Convención de Derechos de las Personas con Deficiencia, Decreto 6.949/2009 (BRASIL, 2009), defiende: a) el aprendizaje de niños sordos utilizando la lengua de signos, garantizando así su accesibilidad; b) la facilitación del aprendizaje de 
http://dx.doi.org/10.5902/1984686X48495

la lengua de signos y promoción de la identidad lingüística de la comunidad sorda; c) garantizar que la educación de personas, en particular niños ciegos, sordociegos y sordos, sea realizada en las lenguas y en los modos y medios de comunicación más adecuados al individuo y en ambientes que favorezcan al máximo su desarrollo académico y social (BRASIL, 2009, artículo 24).

Así, la necesidad de ofrecer a los alumnos sordos clases cuya lengua de instrucción sea la lengua de signos es evidente, puesto que es imposible que un profesor fluente en lengua de signos y en portugués oral pueda usar simultáneamente dos lenguas para propósitos pedagógicos. Si los niños sordos son incluidos en clases con alumnos oyentes el trabajo del profesor bilingüe consistirá en una traducción, y es sabido que durante los años iniciales el hecho de que los niños no sean competentes en lengua de signos no se resuelve con la traducción (LODI; LACERDA., 2009; LACERDA, 2016).

Por todo ello es fundamental diseñar prácticas para el desarrollo de la lengua de signos en el contexto escolar, principalmente en las aulas en las cuales la lengua de instrucción es la lengua de signos, desde la perspectiva de la Metodología Conversacional (GRÀCIA et al., 2017; GRÀCIA et al., 2015a). Los resultados de las investigaciones realizadas por este equipo ponen de relieve que se observan situaciones en el aula en las que el uso de esta metodología promueve la reflexión sobre el lenguaje utilizado por los alumnos, la autoregulación de los turnos, la participación activa, así como otras, que facilitan tales procesos gracias a la participación de los docentes en un proceso de desarrollo profesional con la aplicación digital EVALOE-SSD.

En este contexto, el objetivo de la investigación que se presenta fue aplicar la EVALOE-SSD (GRÀCIA et al., 2018) en una escuela ordinaria que desarrolla un proyecto de educación inclusiva bilingüe con alumnos sordos como proceso de formación profesional de una maestra bilingüe en el uso y promoción de la lengua de signos brasileña (Libras) con sus alumnos. La intención era ayudarle a profundizar en la Metodología Conversacional, que está en la base de la EVALOE-SSD en su versión adaptada, teniendo en cuenta las características de las lenguas de signos.

Entendemos que la formación continuada de maestros bilingües con la aplicación digital EVALOE-SSD puede contribuir a la profundización del conocimiento sobre el uso de esta lengua como lengua de instrucción, para prácticas de desarrollo del lenguaje por parte de los alumnos. 
http://dx.doi.org/10.5902/1984686X48495

\section{Método}

\section{Participantes}

Una docente de educación infantil que trabaja en una escuela como maestra bilingüe. Es oyente, pero domina la lengua de signos puesto que ha participado en formación continuada en lengua de signos en la escuela y en otras prácticas sociales. Es tutora de educación infantil desde hace 11 años y tiene ocho años de experiencia como maestra bilingüe con alumnos sordos. En su grupo clase la lengua de instrucción es la lengua de signos y sus alumnos sordos son una niña (A1) y un niño (A2) de seis años, y un niño de 2 años (Aa).

Quadro 1. Alumnos participantes de Educación Infantil.

\begin{tabular}{|l|l|l|}
\hline Identificación & $\begin{array}{l}\text { Inicio en la Educación } \\
\text { Inclusiva Bilingüe }\end{array}$ & Edad al inicio de la recogida de datos \\
\hline $\mathrm{Aa}$ & $03 / 2017$ & 2a y $9 \mathrm{~m}$ \\
\hline $\mathrm{A} 1$ & $09 / 2017$ & $6 \mathrm{a}$ $2 \mathrm{~m}$ \\
\hline $\mathrm{A} 2$ & $10 / 2015$ & $6 \mathrm{y}$ y $2 \mathrm{~m}$ \\
\hline
\end{tabular}

Fuente: elaboración propia (2020).

Participó también un equipo de investigación compuesto por la investigadora, y dos estudiantes de grado que hicieron las grabaciones de tres sesiones de clase en vídeo y las evaluaran conjuntamente, con la aplicación digital EVALOE-SSD.

\section{Instrumentos}

\section{Aplicación Digital EVALOE-SSD}

El principal instrumento utilizado en esta investigación ha sido la aplicación digital EVALOE-SSD (GRÀCIA et al., 2018) en su versión para contextos de uso de lengua de signos. La maestra lo ha utilizado para aprender una metodología, para autoevaluar sus clases en relación con esta metodología, para tomar conciencia de sus clases, y para tomar decisiones de mejora. El equipo de investigación lo ha utilizado para evaluar 3 de las sesiones de clase de la maestra con sus alumnos y para hacer el seguimiento de las autoevaluaciones de la maestra.

Con el objetivo de describir el funcionamiento de la aplicación digital, a continuación se detallan los pasos que tiene que hacer la maestra para utilizar la EVALOE-SSD: 1) Después de haber proporcionado su correo electrónico a la investigadora, la maestra recibe un correo de alta como usuaria de la aplicación; 2) Una vez entra en la aplicación, lo que 
encontrará inicialmente es una pantalla que la llevará directamente a un Tutorial, que le sugiere que haga una autoevaluación semanal de una de sus clases; 3) Cuando entra en la aplicación le aparecen tres ventanas emergentes en las que se explica qué es EVALOESSD, finalidades y características, y las cinco dimensiones en las que están agrupados los 30 ítems; 4) En la pantalla de inicio visualizará el enlace "Responder cuestionario", y tendrá la opción de hacer una autoevaluación, asignando el color verde (muy satisfactorio), naranja (satisfactorio) o rojo (poco satisfactorio) a cada uno, según sus acciones y las de los alumnos en la clase evaluada se ajusten a lo que se afirma en el ítem.

Se visualizarán los 30 ítems de uno en uno, cada uno en una pantalla, y la maestra tendrá la opción de seleccionar cada ítem el enlace: "¿Qué puedo hacer para incorporar esta acción en mi práctica docente?" donde encontrará una ayuda en formato texto (preguntas de reflexión para ayudarla a entender el ítem, a evaluarlo y a introducirlo en sus clases) para todos los ítems, y solo algunos tendrán otras ayudas en formato vídeo, audio o imagen. Al finalizar el cuestionario verá un cuadro-resumen con sus respuestas a los ítems, agrupados por dimensiones. En la pantalla siguiente, aparecerán seis ítems y se le pedirá que tome una decisión, que consistirá en elegir tres. Esto supone que tratará de incorporar estas tres acciones (suyas o de sus alumnos) a durante las clases siguientes, hasta la próxima autoevaluación.

\section{Cuestionario de Valoración Final del EVALOE-SSD}

Se ha utilizado esta herramienta para recoger la percepción de la maestra participante sobre la aplicación EVALOE-SSD. EI Cuestionario consta de 57 preguntas, tanto abiertas como cerradas, que están agrupadas en cuatro partes: 1) Información sobre la aplicación digital EVALOE-SSD; 2) Redacción de cada ítem respecto a su claridad, idoneidad/pertinencia, importancia y facilidad para escoger una de las tres respuestas; 3 ) Toma de decisiones; y 4) Uso de la aplicación digital y su valoración para la mejora de la práctica docente.

\section{Procedimiento}

El proyecto fue aprobado por el Comité de Ética de la universidad a la que pertenece una de las investigadoras.

Los padres/tutores de los alumnos sordos y maestra participaron en una reunión en la cual se presentó la EVALOE-SSD y los objetivos de la investigación. Después de todas las 
http://dx.doi.org/10.5902/1984686X48495

aclaraciones en relación con los objetivos, riesgos y garantía de anonimato, fueron firmados los Consentimientos informados, de acuerdo con los criterios de la Resolución 466/2012 del Consejo Nacional de Salud.

Las fases que se siguieron en la recogida de datos fueron las siguientes: a) Se registró una sesión de clase (primera sesión) en la que estaban presentes la maestra y los tres alumnos, que fue evaluada por el equipo de investigación, y también por la maestra. El número de ítems que tiene el primer cuestionario que contesta la maestra depende de la evaluación que las investigadoras han realizado de la primera sesión observada. Progresivamente van apareciendo más ítems. En la evaluación número 5 ya aparecen los 30 ítems. Se le explicó que a partir de aquel momento debería intentar pensar en una de sus clases semanales con los tres alumnos y autoevaluarla con la EVALOE-SSD; b) La maestra continuó utilizando la EVALOE-SSD para autoevaluar sus clases, para tomar decisiones durante seis semanas; c) Se registró una nueva sesión de clase (segunda sesión) en la que estaban presentes la maestra y los tres alumnos, que fue evaluada por el equipo de investigación; d) La maestra continuó utilizando la EVALOE-SSD durante cinco semanas más; e) Se registró una última sesión de clase (tercera sesión) en la que estaban presentes la maestra y los tres alumnos, que fue evaluada por el equipo de investigación; f) se pidió a la maestra que contestase el cuestionario de valoración final.

\section{Resultados}

\section{Resultados descriptivos de las sesiones observadas}

En la primera sesión observada la maestra inició la actividad mostrando las imágenes a los alumnos (ver Figura 1), proponiéndoles que contaran lo que estaban viendo. La primera alumna que tomó la iniciativa fue A1 que, juntamente con la profesora, narró la historia, a continuación, A2 hizo lo mismo. Después de esa narración, la profesora decidió contar la historia, viñeta por viñeta, preguntando a los alumnos lo que estaba ocurriendo en cada una de ellas, tratando de aclarar algunos aspectos de la historia. 
http://dx.doi.org/10.5902/1984686X48495

Figura 1 - llustración utilizada en la primera sesión observada (La Bruja, el perro y el gato)

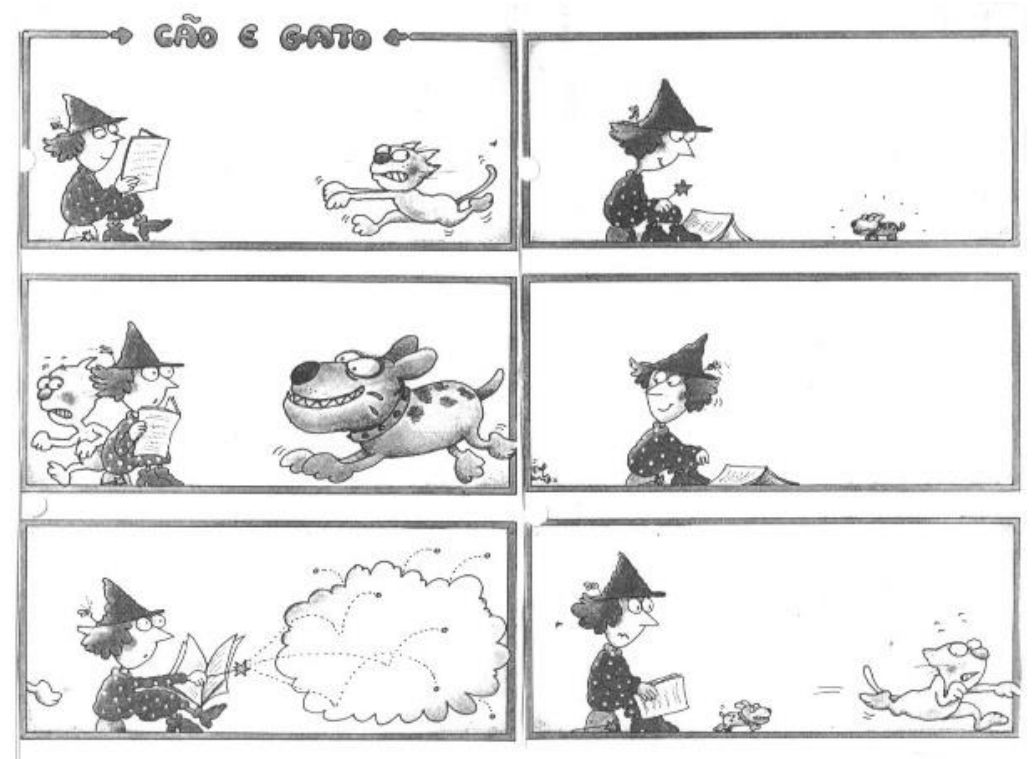

Fuente: FURNARI (2013).

Terminada esta parte de la tarea, les preguntó qué les parecía que pasó después de la última imagen, concretamente les pidió que decidieran y explicaran el desenlace entre todos. Sin embargo, los alumnos sólo contestaron las preguntas que les formuló directamente la maestra, esto es, no dialogaron entre sí, ni regularon su conversación. Los alumnos se mostraron bastante dependientes de la gestión de la docente, no demostrando iniciativas de turnos discursivos o proposición de temas.

En la segunda sesión observada la maestra separó unas piezas de ropa en grupos (pantalones, camisas, medias, gorros) y muñecos, e inició la actividad explicando a los alumnos la propuesta. Inicialmente solamente los alumnos mayores (A1 y A2) participaron, y mientras ellos escogían las piezas y vestían los muñecos, el alumno más pequeño (Aa) permanecía ajeno. Sin embargo, a lo largo de la actividad él se aproximó y comenzó la interaccionar con la maestra, mostrando interés por la actividad. Durante esta actividad fue posible observar los alumnos mayores interaccionando con la maestra y entre sí, asumiendo algunos turnos discursivos sin necesitar la gestión de la maestra. Aunque sus preguntas todavía fueron fundamentales para el desarrollo de la actividad.

En la tercera y última sesión observada la maestra escogió trabajar con los alumnos una situación de juego utilizando frutas y verduras de plástico. Cada alumno tenía una cesta y la profesora les propuso reconocer los alimentos que consumían o les gustaban, y a buscar la parte que faltaba del alimento (por ejemplo, piezas con velcro, de forma que para montar una piña tenían que juntar las dos partes). Durante el juego fueron construyendo un 
http://dx.doi.org/10.5902/1984686X48495

pequeño diálogo, preguntando al compañero sobre lo que más le gustaba, etc. En esta sesión participaron los tres niños en la actividad, interaccionando entre ellos y con la maestra, mostrando iniciativa discursiva en diversos momentos.

\section{Autoevaluaciones de la maestra}

La maestra utilizó la EVALOE-SSD (GRÀCIA et al., 2018) para evaluar sus clases cada 10 o 15 días utilizando su teléfono móvil. En sus autoevaluaciones demostró tener buen dominio de algunas estrategias y sus alumnos también de algunas competencias desde el inicio, y en otros casos la competencia fue mejorando a lo largo del proceso de formación con la herramienta digital. La maestra autoevaluó su práctica docente asignando, en la mayoría de casos, el color naranja (satisfactorio) y verde (muy satisfactorio) a los ítems, valorando en rojo (poco satisfactorio) solamente siete de ellos. Se observa que la maestra valora en rojo el ítem 'actitud activa de los alumnos durante las actividades', en dos ocasiones de las siete evaluaciones. No todos los ítems fueron contestados el mismo número de veces porque no todos aparecieron desde el inicio. En general hay un incremento en las valoraciones de las sesiones de clase evaluadas a lo largo del proceso de formación.

\section{Evaluaciones del equipo de investigación}

La EVALOE-SSD permite contrastar las valoraciones del equipo de investigación y las de la maestra. Cuando la maestra rellena la EVALOE-SSD se aprecia que en la valoración de los ítems hay cierta convergencia en cuanto a las valoraciones en rojo para las dimensiones de 'funciones comunicativas de los alumnos' y 'gestión de la conversación por parte de los alumnos'.

Sin embargo, se percibe la divergencia de valoración en dos ítems de la dimensión 'diseño instruccional', puesto que la maestra valora en verde 4 veces el ítem 'revisamos los conocimientos previos que tenemos de la lengua de signos' (de las 5 veces que aparece) y 6 veces 'explicito los objetivos relacionados con la lengua de signos' (de las 6 veces que aparece), mientras el equipo investigador valora el ítem en rojo en dos ocasiones, y en tres el segundo.

En la Figura 2 se perciben otras diferencias, como es el hecho de que para la maestra sus acciones se van valorando cada vez mejor (línea azul), mientras que la valoración por parte del equipo de investigación (línea naranja) en las dos primeras evaluaciones fue baja, 
http://dx.doi.org/10.5902/1984686X48495

y mejoró en el último registro. Todo ello a pesar de que en las cuatro primeras autoevaluaciones de la maestra el cuestionario tenía menos de 30 ítems.

Figura 2 - Puntuaciones de la maestra (línea azul) y del equipo de investigación (línea naranja) ${ }^{1}$

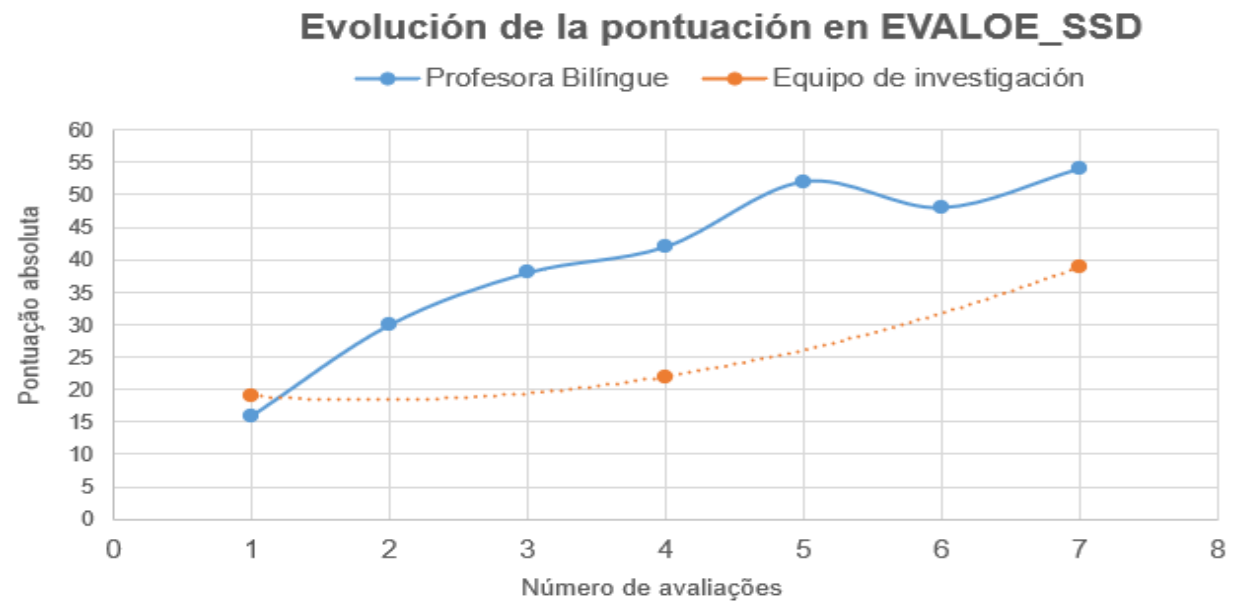

Fuente: GRÀCIA et al. (2018). Aplicación Digital EVALOE-SSD.

\section{Percepción de la maestra del proceso de formación}

Al final del proceso de formación la maestra contestó un cuestionario de valoración de la experiencia. Respecto a la aplicación digital, consideró que la primera vez que entró en la aplicación le resultó 'fácil', y que el tutorial era 'claro', puesto que le ayudó a sentirse segura para usar la aplicación. Sin embargo, le pareció 'complicado' su uso en la etapa de educación infantil, ya que "algunos aspectos eran difíciles de entender, o por la edad de los niños (...) no era posible aplicar las sugerencias".

En relación con la redacción de los ítems y de su claridad, idoneidad/pertinencia e importancia, la maestra señaló que le pareció 'algo confusa y complicada' la redacción referente a algunos ítems: "Algunos términos son desconocidos, o no estaban bien traducidos (del español al portugués)". Y las preguntas de reflexión incluidas en las ayudas en formato de texto vinculadas a cada uno de los ítems, fueron consideradas 'útiles' y las utilizó 'algunas veces'.

Sobre la percepción del objetivo de la aplicación, la maestra consideró que era una 'herramienta de formación para autoevaluar y mejorar la práctica docente referente a la enseñanza de la lengua de signos'.

La maestra señaló que la aplicación había sido 'importante para poder valorar globalmente lo que se realizaba en las clases y promover el desarrollo de la competencia 
comunicativa de los alumnos', en 10 de los 30 ítems de la escala. Los ítems considerados 'más importantes' por la maestra fueron que 'los alumnos autoevaluasen su comportamiento comunicativo en relación con los objetivos de la lengua de signos' y que los alumnos tuviesen una 'actitud activa durante las actividades'. Además, señaló que 9 de los ítems le parecían 'fáciles' de contestar, la mayoría de ellos vinculados con la dimensión de 'funciones comunicativas y estrategias de la docente'. De los seis ítems caracterizados como 'pertinentes', dos se refieren directamente al uso de la lengua de signos, presentes en la dimensión de 'diseño instruccional'.

En relación con el uso de la herramienta, la maestra consideró 'pertinentes y fáciles' las seis opciones ofrecidas para la toma de decisiones. Sobre la experiencia del uso de la herramienta, señaló la opción 'claramente un enriquecimiento, aprendizaje y mejora de mi práctica docente vinculada a la competencia comunicativa' para definir el resultado del proceso del uso de la EVALOE-SSD. Finalmente, la maestra indicó que como media había dedicado '15 minutos' a hacer cada autoevaluación, y consideró 'difícil' encontrar un tiempo semanal dedicado al uso de la herramienta, ya que no lo hacía durante su actividad laboral en la escuela.

\section{Discusión}

El objetivo de la investigación fue aplicar la EVALOE-SSD en una escuela ordinaria que desarrolla un proyecto de educación inclusiva bilingüe con alumnos sordos como proceso de formación profesional de una maestra bilingüe en el uso y promoción de la lengua de signos (Libras) con sus alumnos.

De forma general, la aplicación de la versión EVALOE-SSD adaptada al contexto de educación de sordos se ha mostrado adecuada para su uso por parte de una maestra en relación con la educación bilingüe. Las valoraciones de la maestra y el equipo investigador con la EVALOE-SSD ponen de relieve que en general su uso ha favorecido las interacciones dialógicas en el aula, dando protagonismo a los alumnos $y$, consecuentemente, favoreciendo el desarrollo de la lengua de signos.

En relación con los resultados obtenidos respecto a la dimensión de diseño instruccional, la maestra parece considerar la lengua de signos como una competencia que es necesario desarrollar. Lo cual supone que los objetivos pedagógicos, contenidos, actividades y evaluaciones, además de incluidos en la programación de cada asignatura, 
deben ser explicitados en clase. Los resultados van en la misma dirección que los obtenidos en investigaciones previas con la aplicación digital (GRÀCIA et al., 2015, 2020a).

En esta dimensión, la maestra demostró un progreso continúo en los seis ítems, partiendo de una autoevaluación 'satisfactorio' y alcanzando una valoración de 'muy satisfactorio' en cuatro de los seis ítems incluidos. Sin embargo, las evaluaciones del equipo de investigación indican que en ningún momento tuvo lugar una explicitación por parte de la maestra, de manera clara y detallada, de los objetivos relacionados con la lengua de signos, referente al ítem 'explicito los objetivos relacionados con la lengua oral'. Estos resultados coinciden con otros hallados en investigaciones anteriores con maestras españolas (GRÀCIA et al., 2019, 2020b). La maestra explicó estos resultados en base al hecho de que algunos ítems eran muy difíciles de aplicar en la etapa de educación infantil.

Sin embargo, la evaluación, de forma general, indica reflexión y aprendizaje de la maestra durante el uso del instrumento, como es el caso del ítem vinculado a las propuestas de 'actividades que faciliten trabajar los objetivos de la lengua de signos', que inicialmente es valorado como 'satisfactorio' y al final de la formación como 'muy satisfactorio'. Así, la herramienta parece haber ayudado a la maestra a pensar, reflexionar y considerar puntos que anteriormente no eran el centro de su atención y de sus intenciones pedagógicas.

Coincidimos con Gràcia (2018) en que el hecho de que la maestra revise con sus alumnos los contenidos y objetivos trabajados, señalando aquello que ya dominan en relación con la lengua de signos, contribuye a que ellos tomen conciencia de lo que han aprendido, promoviendo también el desarrollo de su capacidad metalingüística.

En relación con la dimensión vinculada a la 'gestión de la conversación por parte de la docente', la maestra demostró cierta oscilación en las respuestas. Respecto al ítem 'doy tiempo a los alumnos para que tomen su turno', alterna dos valoraciones durante las autoevaluaciones, de manera que en determinados momentos considera su práctica coherente y 'muy satisfactoria', y en otros la valora como 'satisfactoria'. La variabilidad de las respuestas demuestra que percibe la necesidad de introducir algunos ajustes respecto a la gestión de la conversación para que esta sea plenamente satisfactoria. Dar el tiempo adecuado para que los alumnos sordos, con poco dominio de la lengua de signos, asuman el turno comunicativo y se expresen, no siempre es fácil, en función de la edad, de la atención de los compañeros y de la manera como la maestra gestiona las preguntas.

La tercera dimensión supone que el aula sea un espacio abierto de diálogo en el cual la conversación y el discurso de manera general puedan ser instrumentos para el 
aprendizaje de los diversos contenidos escolares. Para que eso ocurra, es necesario que los alumnos puedan regular su propia conversación, sin que la docente gestione siempre los turnos, lo cual supone crear espacios de autonomía discursiva por parte de los alumnos.

En ese sentido, la maestra demostró dificultades para incorporar estrategias de modo que los alumnos consiguiesen gestionar sus turnos comunicativos y tuvieran una actitud activa durante las actividades. Tanto en las evaluaciones del equipo de investigación como en las autoevaluaciones, las valoraciones oscilaron entre las tres opciones posibles, revelando dificultades para conseguir este objetivo en las clases, tanto por parte de la maestra como de los alumnos. Un factor que probablemente influyó en este resultado es la edad de los niños y el dominio restringido de la lengua de signos. El aprendizaje tardío de la lengua de signos puede comprometer la autonomía discursiva, dadas las experiencias restringidas con la lengua en diferentes esferas sociales (MOURA, 2013).

En los ítems de la dimensión 'funciones comunicativas y estrategias de los docentes', nuevamente la maestra demostró variaciones en sus valoraciones, principalmente en relación con el ítem 'expando los enunciados de los alumnos'. De modo general, esta dimensión recoge estrategias vinculadas al grado en el que la maestra recoge las aportaciones de los alumnos y las expande, lo que supone un modelo más elaborado de expresión en lengua de signos.

Coincidiendo con las evaluaciones del equipo de investigación, la maestra evolucionó mucho durante el proceso, especialmente en el ítem 'enseño fórmulas de interacción social', que al inicio fue valorado como 'poco satisfactorio', en la segunda evaluación como 'satisfactorio' y finalmente obtuvo la máxima valoración.

Esos resultados demuestran la reflexión de la maestra en relación con su práctica, modificando sus fórmulas y métodos para enseñar sus alumnos, considerándolo como uno de los aspectos clave de la enseñanza de la lengua durante las actividades (GRÀCIA et al., 2015a).

La dimensión vinculada a las 'funciones comunicativas de los alumnos', pretende valorar el grado en el que las estrategias de la docente se reflejan en las acciones de los alumnos. De manera que, si el docente no enseña explícitamente a los alumnos 'cómo obtener informaciones' o cómo 'sintetizar y extraer conclusiones', estos no podrán utilizar la lengua de signos con estas finalidades. Nuevamente la maestra presenta cierta falta de seguridad en relación con estos ítems, ya que se aprecia mucha variabilidad en sus valoraciones. 
http://dx.doi.org/10.5902/1984686X48495

De modo general, se puede afirmar que la maestra modificó sus acciones y estrategias, y también las de sus alumnos, en todas las dimensiones, lo cual indica que la propuesta de cambios planteada por Gràcia et al. (2018) en la primera versión de la EVALOE-SSD para adaptarla al contexto de enseñanza de lengua de signos fue adecuada y permitió ayudar a esta docente, y también a otra docente sorda en España (GRÀCIA et al., 2019, 2020a), provocando reflexiones que han contribuido a modificar su práctica pedagógica y la introducción de actividades de interacción con y para mejorar el aprendizaje y uso de la lengua de signos. Se destaca la presencia de textos escritos, vídeos, imágenes, dibujos, recuadros, entre otras (con sugerencias de actividades, preguntas y acciones para ser realizadas en clase) para cada uno de los 30 ítems (GRÀCIA, 2018; GRÀCIA et al., 2020a), como ayudas ofrecidas por la herramienta, favoreciendo la toma de decisiones en relación con su práctica de enseñanza.

La propuesta digital se mostró clara, las ayudas y la forma de presentación de las preguntas se mostraron suficientes y las ayudas de distinto tipo eficaces para que la maestra los usara como herramienta de desarrollo profesional.

Es posible afirmar que, de modo general, las maestras, tanto en Brasil como en España (GRÀCIA et al., 2015b, 2019, 2020a) mejoraron sus valoraciones durante su participación en el proceso de desarrollo profesional utilizando la EVALOE-SSD.

Tanto la docente bilingüe brasileña como la española (GRÀCIA et al., 2019, 2020a) partieron de una autoevaluación inicial baja y progresivamente se observan mejores valoraciones, indicando probablemente no un cambio radical de sus actitudes y estrategias en el aula con sus alumnos, pero sí una mayor atención a cuestiones relativas a la promoción de cambios que promuevan más diálogo en lengua de signos en el ambiente escolar.

Ello supone que la valoración de las clases con intención reflexiva presenta un aumento de la conciencia de las prácticas docentes, incorporando nuevas acciones, corroborando los resultados de otras investigaciones que demuestran la importancia de la formación continuada para profesores (VEGA; GRÀCIA, 2016).

Cabe resaltar que la maestra bilingüe brasileña tuvo la oportunidad de realizar siete auto evaluaciones utilizando la EVALOE-SSD (durante tres meses), mientras que la profesora bilingüe española realizó 18 autoevaluaciones (durante seis meses) (GRÀCIA et al., 2020a). Sin embargo, aunque las dos maestras mejoraran sus valoraciones a lo largo del periodo de uso de la aplicación, ninguna de ellas se aproximó a la puntuación máxima 
posible. Aunque el objetivo de la EVALOE-SSD no sea en absoluto alcanzar la puntuación máxima en un determinado periodo de tiempo, sí pretende proporcionar el esfuerzo reflexivo, de manera que las puntuaciones obtenidas por las maestras pueden indicar que el tiempo de uso de la aplicación (el periodo de formación continuada) ha sido insuficiente para los posibles beneficios que podría proporcionar, si fuera utilizada durante un periodo más extenso.

Así, se ha construido una herramienta digital de desarrollo profesional para ayudar los docentes a mejorar su práctica en relación con la enseñanza de las lenguas de interacción cara a cara. La mayor parte de los docentes que hicieron uso de la herramienta (GRÀCIA et al., 2020a), la usaron de manera correcta y sistemática, autoevaluando sus clases y tomando decisiones e introduciendo cambios en sus actividades de enseñanza. Las puntuaciones de las evaluaciones y autoevaluaciones en todos los casos son más altas a final del periodo de formación, indicando que el profesorado y el alumnado mejoraron sus formas de interacción dialógica mediadas por la lengua oral (o de signos) (GRÀCIA et al., 2019, 2020a). Destacase la imposibilidad de comparar la EVALOE-SSD con otros instrumentos semejantes porque los que hay son muy diferentes en sus propósitos. Por ahora, solo podemos comparar los resultados alcanzados con las investigaciones que hemos realizado nosotros con la propia EVALOE-SSD. A medida que se vaya conociendo más la aplicación digital y otros investigadores presenten resultados, podremos ampliar las discusiones.

\section{Conclusiones}

El objetivo fundamental de la investigación se ha alcanzado en el contexto de educación Infantil. Persiste el interés en realizar un estudio semejante en el contexto de educación Primaria, como ya se ha realizado con maestras españolas (GRÀCIA et al., 2020b), tratando de perfeccionar la herramienta y analizar también su impacto en ese nivel de enseñanza.

Las dimensiones del trabajo docente y también de la EVALOE-SSD, destacan aspectos relevantes, entendiendo la importancia del aprendizaje en cual el alumno es el protagonista, y en cuyo proceso están implicados factores afectivos, emocionales, sociales y cognitivos. De este modo, el análisis realizado demostró la existencia de reflexiones de la maestra participante durante las autoevaluaciones, que ayudaron en su trabajo con la lengua de signos y favorecieron la interacción entre sus alumnos usando signos. 
En este sentido, la afirmación de muchos autores según la cual la escuela es un contexto que promueve el desarrollo del lenguaje (HAMRE, et al., 2014; HOWES et al., 2008) y la necesidad de ayudar a la escuela y a los docentes en la promoción del desarrollo de lenguaje, y en la búsqueda de crear un ambiente favorable para la expresión espontánea para el niño (MERCER; WARWICK; AHMED, 2017), ha sido posible gracias a la herramienta EVALOE-SSD.

Entendemos que es fundamental aprovechar las competencias que ya posee el docente, pero que en ocasiones quedan enmascaradas en la rutina diaria, como es la importancia de la interacción utilizando la lengua de signos, puesto que la mejora de la competencia en esa lengua favorece en los alumnos sordos mayor progreso en el conjunto de las disciplinas curriculares, dado que todas están mediadas por los conocimientos en lengua de signos. Esta también tiene relevancia social, contribuyendo no sólo al desarrollo cognitivo de la persona, sino también a su vida en sociedad y al alcance de sus derechos como ciudadanos. Es por ello que las reflexiones propiciadas por el uso de la EVALOESSD indican que es un instrumento potencialmente eficiente para la formación continuada de profesores, en la línea de mejora constante de la calidad de enseñanza, lo cual tiene un impacto positivo también para los estudiantes oyentes y sordos.

\section{Referências}

BRASIL. Decreto № 5.626. Regulamenta a Lei oㅜ 10.436, de 24 de abril de 2002, que dispõe sobre a Língua Brasileira de Sinais - Libras, e o art. 18 da Lei oㅜ 10.098, de 19 de dezembro de 2000. Publicado no Diário Oficial da União em 22/12/2005.

BRASIL. Decreto № 6.949. Promulga a Convenção Internacional sobre os Direitos das Pessoas com Deficiência. Publicado no Diário Oficial da União em 25/08/2009.

CLARKE, Sherice N., RESNICK, Lauren B., PENSTEIN ROSÉ, Carolyn, Dialogic instruction: A new frontier. En: Lyn CORNO \& Eric ANDERMAN (Eds.), Handbook of educational psychology. 3rd edition (p. 278-388). Mahwah, NJ: Erlbaum, 2016.

GRÀCIA, Marta, CASANOVAS, Josep, SANCHO, Maria R., CASANOVAS, Jordi, CUATRECASAS, Marta, Empowering teachers' self assessment of their own practice on student's oral skills: EVALOE Decision Support System. En: Proceedings of the 10th International Conference on Computer Supported Education: CSEDU, v. 2, p. 272277, 2018. DOI: https://doi.org/10.5220/0006785902720277 
GRÀCIA, Marta, La EVALOE-SSD como herramienta de autoevaluación y de soporte a la toma de decisiones docentes para convertir las aulas de educación infantil y primaria en entornos comunicativos. En M. T. Signes, C. Carreira Zafra, M. Kazmierczak (eds.), EI rol central de la narración en el contexto educativo (197-217). Vigo: Editorial Academia del Hispanismo, 2018.

GRÀCIA, Marta, CASANOVAS, Josep, RIBA, Carles, SANCHOA, Maria R., JARQUE, M. Jopep, CASANOVAS, Jordi, VEGA, Fàtima. Developing a digital application (EVALOEDSS) for the professional development of teachers aiming to improve their students' linguistic competence, Computer Assisted Language Learning, 2020a DOI: https://doi.org/10.1080/09588221.2019.1707690

GRÀCIA, Marta, GALVÁN-BOVAIRA, M. José, SÁNCHEZ-CANO, Manel, Análisis de las líneas de investigación y actuación en la enseñanza y el aprendizaje del lenguaje oral en contexto escolar. Revista Española de Lingüística Aplicada, v. 30 n. 1, p. 188-209, 2017.

GRÀCIA, Marta (Coord.), GALVÁN-BOVAIRA, M. José, SÁNCHEZ-CANO, Manel, VEGA, Fàtima, VILASECA, Rosa, y RIVERO, Magdalena, Valoración de la enseñanza de la lengua oral. Escala EVALOE. Barcelona: Graó, 2015a.

GRÀCIA, Marta, GALVÁN-BOVAIRA, M. José, VILASECA, Rosa, RIVERO, Magdalena, SÁNCHEZ-CANO, Manel, El cambio conceptual de dos maestras en relación con la enseñanza y el aprendizaje de la lengua oral a través de un asesoramiento: un estudio de casos. Revista de Logopedia, Foniatría y Audiología, v. 32 n. 4, p.179-189, 2012.

GRÀCIA, Marta, De LACERDA, Cristina B. F., JARQUE, M. Josep, DOMENICONI, Camila, CASANOVAS, Josep, SANCHO, M. Ribera, CASANOVAS, Jordi, CUATRECASAS, Marta, Diseño y construcción de un Sistema de Soporte a la toma de Decisiones de los docentes sobre las interacciones comunicativas y lingüísticas en el aula. Multidisciplinary Journal of School Education v. 2 n. 16, p. 57-83, 2019.

GRÀCIA, Marta, JARQUE, M. Josep, RIBA, Carles, VEJA, Fàtima, Uso de una herramienta digital como recurso de desarrollo profesional para mejorar la competencia comunicativa oral de alumnos de educación infantil y primaria. Revista de investigación en logopedia, v. 10 n.2, p. 137-151, 2020b.

GRÀCIA, Marta, VEGA, Fàtima, GALVÁN-BOVAIRA, M. José, Developing and testing EVALOE: a tool for assessing spoken language teaching and learning in the classroom. Child Language Teaching Therapy, v. 31 n. 3, p. 287-304, $2015 \mathrm{~b}$.

HAMRE, Bridget, HATFIELD, Bridget, PIANTA, Robert., y JAMIL, Faiza. Evidence for general and domain-specific elements of teacher-child interactions: associations with preschool children's development. Child Development, v 85, n39, p.1257-1274, 2014.

HOWES, Carollee, BURCHINAL, Margaret, PIANTA, Robert, BRYANT, D., EARLY, D., CLIFFORD, R., y BARBARIN, Oscar Ready to Learn? Children's Pre-Academic Achievement in Pre-Kindergarten Programs. Early Childhood Research Quarterly, v. 23 n.1, p. 27-50, 2008. 
JONES, Deborah. Talking about talk: reviewing oracy in English primary education. Early Child Development and Care, v.187 n.3/4, p. 498-508, 2017.

KORTHAGEN, Fred. A. J. . Teacher reflection: What it is and what it does. Em: Edward G. PULTORAK (Ed.). The purposes, practices, and professionalism of teacher reflectivity: Insights for twenty-first-century teachers and students. Lanham, ML: Rowman \& Littlefield, p. 377- 401, 2010.

LACERDA, Cristina B. F. de. Intérprete de LIBRAS: em atuação na educação infantil e no ensino fundamental. 1. ed. Porto Alegre: Editora Mediação/FAPESP, 2009. v. 1. 95p.

LACERDA, Cristina B. F. de. Bilingual Inclusive Deaf Education: Research in the Public Education System. En: GERNER, Barbara; KARNOPP, Lodenir (Org.). Bilingual Deaf Education and Deaf Culture in Latin America. 1ed.Washington EUA: Gaullaudet Press, 2016, v. 1, p. 50-67.

LACERDA, Cristina.B.F.de y LODI, Ana.C.B. A inclusão escolar bilíngue de alunos surdos: princípios, breve histórico e perspectivas. En: LODI, Ana C.B. e LACERDA, Cristina B.F.de; Uma escola duas línguas: letramento em língua portuguesa e língua de sinais nas etapas iniciais de escolarização. Porto Alegre: Mediação, 2009. p. 11-32.

LACERDA, Cristina B.F. de, GRÀCIA, Marta, y JARQUE, Maria J. Adaptación de una escala de evaluación conversacional para el contexto de educación de alumnos sordos. En: MARTÍN, Mário B. y SEBASTIÁN, Eládio H. (Org.). Hacia un modelo educativo de calidad y transformador. 1ed.Alcalá de Henares Espanã: Fundación Santillana, 2018, v. 1, p. 197-205.

LACERDA, Cristina B.F. de, GRÀCIA, Marta, y JARQUE, Maria J. Línguas de Sinais como Línguas de Interlocução: o Lugar das Atividades Comunicativas no Contexto

Escolar. Revista brasileña de educación especial, 26 (2), 299-312, 2020.

LODI, Ana C.B. y LACERDA, Cristina B.F. de. Uma escola duas línguas: letramento em língua portuguesa e língua de sinais nas etapas iniciais de escolarização. Porto Alegre: Mediação, 2009.

MAYBIN, Janet, MERCER, Neil, y STIERER, Barry Thinking Voices: The work of the National Oracy Project, p. 86-95. Kate Norman, 1992.

MERCER, Neil. The analysis of the classroom talk: Methods and methodologies. British Journal of Educational Psychology, v 80 n1, p. 1-14, 2010.

MERCER, Neil. Why Oracy Must Be in the Curriculum (and Group Work in the Classroom), FORUM, v 57, n 1, p. 67-74, 2015.

MERCER, Neil, WARWICK, Paul y AHMED, A. An oracy assessment toolkit: Linking research and development in the assessment of students' spoken language skills at age 11-12. Learning and Instruction, v 48, p. 51-60, 2017. 
MOURA, Maria Cecília de. Surdez e Linguagem. En: LACERDA, CristinaB.F.de y SANTOS, Lara F. dos. (Org.). Tenho um aluno surdo, e agora? Introdução à Libras e educação de surdos. São Carlos: EdUFSCar, 1ed., p. 13-26, 2013.

PIASTA, Shayne B., JUSTICE, Laura M., CABELL, Sonia Q, WIGGINS, Alice K., PENCE, Kara, y CURENTON, Stephanie. Impact of professional development on preschool teachers' conversational responsivity and children's linguistic productivity and complexity. Early Childhood Research Quarterly, v 27 n 3, p.387-400, 2012.

SILVA QUIROZ, Juan. E. Estándares TIC para la Formación Inicial Docente: una política pública en el contexto chileno. Archivos Analíticos de Políticas Educativas, v $20 \mathrm{n} 7$, 2012. Recuperado el día 25 de mayo de 2020 en http://epaa.asu.edu/ojs/article/view/962.

SHIM, Jung P., WARKENTIN, Merill, COURTNEY, James F., POWER, D. J., SHARDA, R., y CARLSSON, Christer. Past, present, and future of decision support technology. Decision support systems, v 33 n 2, p. 111-126, 2002.

VAN DER VEEN, Chiel, DE MEY, Langa, VAN KRUISTUM, Claudia y VAN OERS, Bert The effect of productive classroom talk and metacommunication on young children's oral communicative competence and subject matter knowledge: An intervention study in early childhood education. Learning and Instruction, v 48, p. 14-22, 2017.

VAN DE POL, Janneke, MERCER, Neil, y VOLMAN, Monique, Scaffolding Student Understanding in Small-Group Work: Students' Uptake of Teacher Support in Subsequent Small-Group Interaction. Journal of the Learning Sciences, v 28 n 2, p. 206-239, 2019.

VEGA, Fàtima, GRÀCIA, Marta, Asesoramiento a docentes en el uso de un Sistema Aumentativo y Alternativo de Comunicación. Revista de Investigación en logopedia, $v$. 6 n. 2, 169-202, 2016.

\section{Notas}

${ }^{1}$ Rojo (0 puntos), naranja (1 punto), verde (2 puntos). La puntuación máxima es 60 (30x2).

\section{Correspondência}

Marta Gràcia - Universidad de Barcelona, Facultad de Psicología, Passeig de la Vall d'Hebrón, 171, Barcelona - Espanha

CEP: 08035

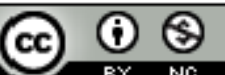

This work is licensed under a Creative Commons Attribution-NonCommercial 4.0 International (CC BY-NC 4.0) 